\title{
INPP1 is differentially expressed in platinum-resistant ovarian cancers.
}

Shahan Mamoor, MS1

1Thomas Jefferson School of Law

San Diego, CA 92901

mamoorsk@tjsl.edu

Ovarian cancer is the most common reason for a gynecological cancer death in the developed world and fifth leading cause of cancer death in women in the United States $(1,2)$.

Chemotherapy includes the use of platinum drugs (3) and resistance to platinum drugs is a serious problem for women diagnosed with ovarian cancer $(4,5,6)$. We found, using two published datasets $(7,8)$ that INPP1 was one of the genes most differentially expressed when comparing the transcriptomes of platinum-resistant and platinum-sensitive tumors and cell lines but that the pattern of differential expression was opposite in cell lines versus that in primary tumors from patients. Manipulation of INPP1 expression should be assessed for its ability to reverse platinum resistance.

Keywords: ovarian cancer, platinum resistance, systems oncology, tumor transcriptome analysis, targeted therapeutics in ovarian cancer, INPP1. 


\section{Introduction}

Ovarian cancer is diagnosed in over 24,000 women each year and in 2018 greater than 17,000 women expired from the disease in the United States (1). Standard treatment of ovarian cancer includes the use of platinum chemotherapies including carboplatin and oxaliplatin (3) in a taxane-platinum combination (9) which frequently leads to resistance and subsequent treatment failure $(4,5,6)$. Understanding how to prevent, delay or reverse this resistance requires a basic understanding of the transcriptional changes that accompany this resistance. We performed whole transcriptome analysis of tumors from platinum-resistant ovarian cancer patients by comparison to the transcriptome of tumors from platinum-sensitive ovarian cancer patients (7). We performed the same global differential gene expression analysis on the IGROV-1 ovarian cancer cell line as well as two platinum-resistant IGROV-1 variants pretreated with oxaliplatin and carboplatin (8). We found that the inositol polyphosphate-1phosphatase INPP1 was among the most differentially expressed genes in the platinumresistant state in ovarian cancer across both datasets. Modulation of INPP1 expression should be assessed for its ability to manipulate the platinum resistance process in a targeted fashion.

\section{Methods}

We used datasets GSE114206 ( $n=6$ for platinum-sensitive and $n=6$ for platinumresistant) and GSE58470 ( $n=3$ for platinum-sensitive and $n=6$ for platinum-resistant) to perform this analysis using GEO2R. The authors of GSE114206 utilized Agilent 026652 Whole Human Genome Microarray 4x44K v2 technology to generate the microarray dataset utilized here. The authors of GSE58470 utilized Illumina HumanHT-12 V3.0 expression beadchip technology to generate the microarray dataset also utilized here. The Benjamini and Hochberg method of $p-$ value adjustment was utilized, and p-value adjustment was set to "Auto-detect". The NCBI generated category of platform annotation was utilized. To evaluate the statistical significance of differences in RNA expression values in platinum-resistant versus platinum-sensitive groups, 
a two-tailed, unpaired t test with Welch's correction was performed to obtain a p-value. Pvalues less than 0.05 were considered statistically significant.

\section{Results}

We used two public datasets to determine what genes were differentially expressed in ovarian cancers that had developed resistance to platinum chemotherapy, or what genes changed the expression the most in ovarian tumors and cancer cells that were platinumresistant versus platinum-sensitive.

INPP1 is among the genes whose expression is most different between IGROV-1 ovarian cancer cells that are platinum-sensitive and platinum-resistant

We compared the transcriptome of the IGROV-1 ovarian cancer cell line to that of carboplatin- and oxaliplatin-resistant IGROV-1 ovarian cancer cells lines. We found that INPP1 was one of the most differentially expressed genes when comparing platinum-resistant and platinum-sensitive ovarian cancer cell lines, ranking 92 in expression out of 48803 transcripts detected and measured in the microarray dataset utilized here.

INPP1 is among the genes whose expression is most different between ovarian tumors that are platinum-sensitive and platinum-resistant

We also compared the transcriptomes of primary ovarian tumors isolated from patients with ovarian cancer that were platinum-sensitive with that from patients that had developed platinum resistance. We found that INPP1 was one of the most differentially expressed genes when comparing platinum-resistant and platinum-sensitive ovarian cancers, ranking 108 in expression out of 29339 transcripts detected and measured in the microarray dataset utilized here. 
INPP1 expression in platinum-resistant cancer cell lines is significantly lower but significantly higher in platinum-resistant patient tumors

We isolated the exact mRNA expression values of INPP1 in the parental IGROV-1 cell line as well as the carboplatin- and oxaliplatin-resistant variants and performed a statistical test to determine if the difference in expression between platinum-resistant and platinum-sensitive cell lines was significantly different. The expression of INPP1 was significantly lower in platinum-resistant ovarian cancer cell lines versus that of platinum-sensitive ovarian cancer cell lines (Figure 1, $p=0.0053$ ).

We then isolated the exact mRNA expression values of INPP1 in each patient's tumor and performed a statistical test to determine if the difference in expression between platinumresistant and platinum-sensitive tumors was significantly different. Contrary to our findings in ovarian cancer cell lines, the expression of INPP1 was significantly higher in platinum-resistant ovarian cancers versus that of platinum-sensitive ovarian cancers (Figure 1, $p=0.0047$ ).

\section{Discussion}

Treatment of ovarian cancer with platinum agents frequently leads to platinum resistance $(4,5,6)$. Understanding the transcriptional nature of this resistance is essential to understanding in a targeted, specific manner how to reverse or delay this resistance in order to increase quality of life and median survival in women with ovarian cancer. We compared the transcriptomes of platinum-sensitive ovarian cancer cell line and platinum-sensitive ovarian tumors to the transcriptomes of platinum-resistant ovarian cancer cell line variants and platinum-resistant ovarian tumors to determine in a systems-level, unbiased manner what genes are expressed most differently in the platinum-sensitive and platinum-resistant states (7, 8). We found that INPP1 expression changed more significantly than greater than $99 \%$ of the rest of the transcriptome in the platinum-resistant state. Though INPP1 was expressed at significantly lower levels in carboplatin and oxaliplatin-resistant cell lines, it was expressed at 
significantly higher levels in platinum-resistant patient tumors. We do not understand the basis for this difference but could reflect "artificial" adaptation by tumor cells in cell culture (in vitro). INPP1 functions to dephosphorylate free inositols that have been polyphosphorylated (10). One study demonstrated that INPP1 was over-expressed in multiple cancer cell lines and expressed at higher levels in higher-grade primary ovarian cancer versus lower-grade ovarian cancers (11). Consistent with this report, we find that in ovarian cancer, INPP1 is expressed at higher levels in platinum-resistant primary ovarian tumors.

Platinum resistance is the cause of treatment failure after chemotherapy with platinumtaxane combinations in ovarian cancer. We identified, by studying the total transcriptome of tumors isolated from women with ovarian cancer, one of the genes whose expression changes the most in tumors after development of platinum resistance: INPP1. INPP1 should be assessed in xenograft mouse models of ovarian cancer (12) for its ability, upon modulation of expression, to control the resistance process in order to develop novel strategies to prevent, delay or reverse platinum resistance in women with ovarian cancer. 


\section{References}

1. Chornokur, G., Amankwah, E.K., Schildkraut, J.M. and Phelan, C.M., 2013. Global ovarian cancer health disparities. Gynecologic oncology, 129(1), pp.258-264.

2. Torre, L.A., Trabert, B., DeSantis, C.E., Miller, K.D., Samimi, G., Runowicz, C.D., Gaudet, M.M., Jemal, A. and Siegel, R.L., 2018. Ovarian cancer statistics, 2018. CA: a cancer journal for clinicians, 68(4), pp.284-296.

3. Markman, M., Rothman, R., Hakes, T., Reichman, B., Hoskins, W., Rubin, S., Jones, W., Almadrones, L. and Lewis Jr, J.L., 1991. Second-line platinum therapy in patients with ovarian cancer previously treated with cisplatin. J Clin Oncol, 9(3), pp.389-393.

4. Cooke, S.L. and Brenton, J.D., 2011. Evolution of platinum resistance in high-grade serous ovarian cancer. The lancet oncology, 12(12), pp.1169-1174.

5. Eckstein, N., 2011. Platinum resistance in breast and ovarian cancer cell lines. Journal of Experimental \& Clinical Cancer Research, 30(1), p.91.

6. Giaccone, G., 2000. Clinical perspectives on platinum resistance. Drugs, 59(4), pp.9-17.

7. Veskimäe K, Scaravilli M, Niininen W, Karvonen H et al. Expression Analysis of Platinum Sensitive and Resistant Epithelial Ovarian Cancer Patient Samples Reveals New Candidates for Targeted Therapies. Trans/ Oncol 2018

8. Arrighetti, N., Cossa, G., De Cecco, L., Stucchi, S., Carenini, N., Corna, E., Gandellini, P., Zaffaroni, N., Perego, P. and Gatti, L., 2016. PKC-alpha modulation by miR-483-3p in platinum-resistant ovarian carcinoma cells. Toxicology and applied pharmacology, 310, pp. 9-19.

9. Raja, F.A., Chopra, N. and Ledermann, J.A., 2012. Optimal first-line treatment in ovarian cancer. annals of Oncology, 23(suppl_10), pp.x118-x127.

10. Inhorn, R.C. and Majerus, P.W., 1987. Inositol polyphosphate 1-phosphatase from calf brain. Purification and inhibition by $\mathrm{Li}+, \mathrm{Ca} 2+$, and Mn2+. Journal of Biological Chemistry, 262(33), pp.15946-15952.

11. Benjamin, D.I., Louie, S.M., Mulvihill, M.M., Kohnz, R.A., Li, D.S., Chan, L.G., Sorrentino, A., Bandyopadhyay, S., Cozzo, A., Ohiri, A. and Goga, A., 2014. Inositol phosphate recycling regulates glycolytic and lipid metabolism that drives cancer aggressiveness. ACS chemical biology, 9(6), pp.1340-1350.

12. Pizer, E.S., Wood, F.D., Heine, H.S., Romantsev, F.E., Pasternack, G.R. and Kuhajda, F.P., 1996. Inhibition of fatty acid synthesis delays disease progression in a xenograft model of ovarian cancer. Cancer research, 56(6), pp.1189-1193. 
Table 1

\begin{tabular}{|c|c|c|c|c|c|c|}
\hline & ID & p-value & $t$ & B & Gene & Gene name \\
\hline \multicolumn{7}{|l|}{$\begin{array}{l}\text { GSE54870 } \\
\text { (cell lines) }\end{array}$} \\
\hline 92 & ILMN_1667239 & $6.55 \mathrm{E}-07$ & $-1.23 \mathrm{E}+01$ & 6.643764 & INPP1 & $\begin{array}{l}\text { inositol } \\
\text { polyphosphate-1- } \\
\text { phosphatase }\end{array}$ \\
\hline \multicolumn{7}{|l|}{$\begin{array}{l}\text { GSE114206 } \\
\text { (patient } \\
\text { tumors) }\end{array}$} \\
\hline 108 & A_32_P44453 & $5.19 \mathrm{E}-04$ & 4.5069959 & 0.03253 & INPP1 & $\begin{array}{l}\text { inositol } \\
\text { polyphosphate-1- } \\
\text { phosphatase }\end{array}$ \\
\hline & & & & & & \\
\hline
\end{tabular}

Table 1: INPP1 is differentially expressed in platinum-resistant ovarian tumors isolated from patients with ovarian cancer and in platinum-resistant ovarian cancer cell lines.

The rank of differential expression globally out of 48803 transcripts (GSE54870) or 29339 transcripts (GSE114206), probe ID, the p-value with respect to global differential expression, $t$, a moderated t-statistic, B, the log-odds of differential expression between platinum-resistant and -sensitive groups, the log fold-change in expression between platinum-resistant and sensitive groups, gene symbol and gene name are shown in this chart (extracted from GEO2R). 
cell lines
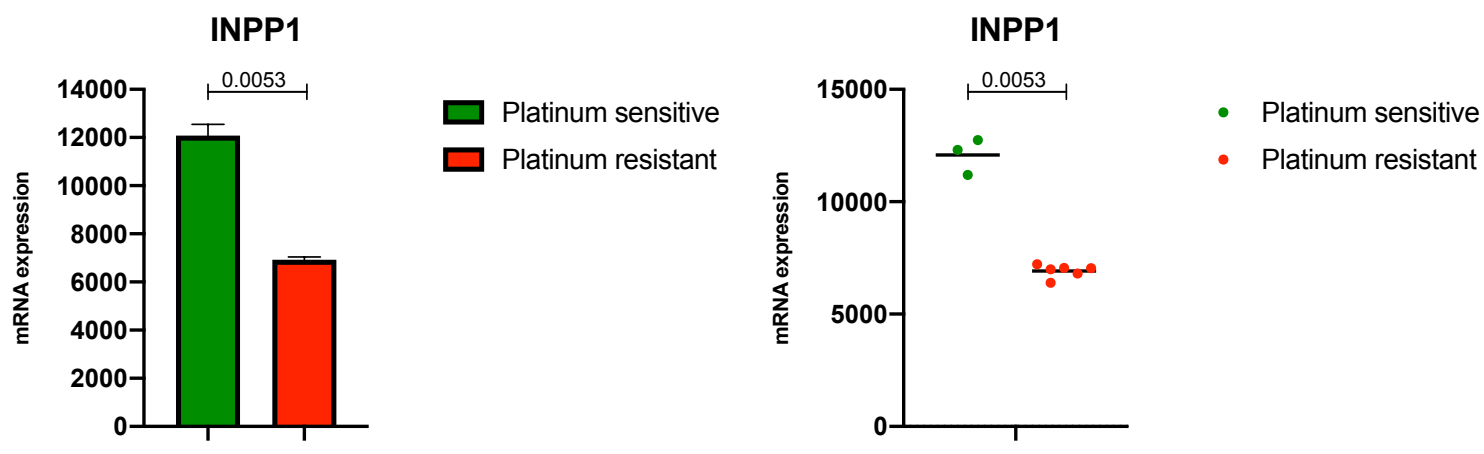

primary tumors

INPP1

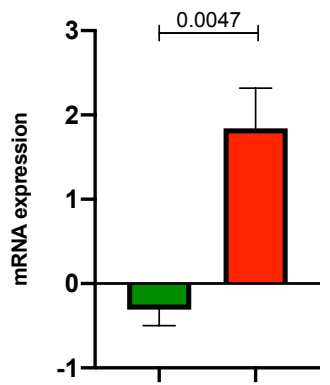

Platinum sensitive

Platinum resistant

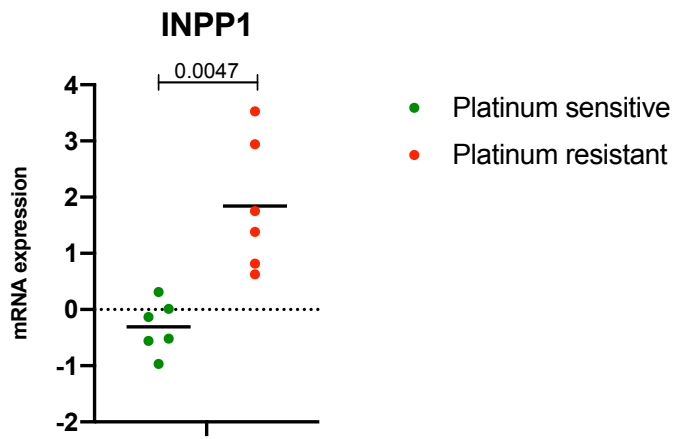

Figure 1: INPP1 is expressed at significantly lower levels in platinum-resistant cell lines but at significantly higher levels in platinum-resistant patient tumors.

The expression of INPP1 in platinum-sensitive (green) and platinum-resistant (red) cell lines (top row) and primary ovarian tumors isolated from patients diagnosed with ovarian cancer (bottom row) is shown by bar graph with the error bars denoting the standard error of the mean (left) and by dots denoting the expression level of INPP1 in each individual cell line replicate or patient tumor (right). A statistical test evaluating the significance of difference in expression between platinum-sensitive and -resistant groups was perfumed using an unpaired, two-tailed $t$ test with Welch's correction and the results of that test, a p-value, is shown on each graph. 